\title{
Another Proliferative Diabetic Retinopathy? A Case Report of Retinal Cavernous Haemangioma Treated with Intravitreal Bevacizumab, Initially Labelled as Persistent Proliferative Diabetic Retinopathy
}

\author{
Myrto Tsagkataki $^{{ }^{*}}$, Ahmad Khalil ${ }^{1}$, Ahmed Kamal ${ }^{1}$ \\ ${ }^{1}$ Ophthalmology Department, Aintree University Hospitals, Liverpool, United Kingdom. \\ Email: ${ }^{*}$ m.tsagkataki@gmail.com
}

Received November $19^{\text {th }}, 2011$; revised December $24^{\text {th }}, 2011$; accepted January $5^{\text {th }}, 2012$.

\begin{abstract}
We present a case of Retinal Cavernous Haemangioma treated with Intravitreal Bevacizumab, which was initially labelled as persistent proliferative diabetic retinopathy with multiple episodes of vitreous haemorrhage. These lesions can be confused with new retinal vessels in diabetics and if correctly diagnosed unnecessary photocoagulation can be avoided. Our patient received a course of three intravitreal Bevacizumab injections $(1.25 \mathrm{mg} / 0.05 \mathrm{ml})$ in order to stop the leakage from the retinal cavernous haemangioma lesions and prevent another episode of vitreous haemorrhage. No intraoperative or postoperative complications were seen. Twenty-two months following treatment there was no recurrence of vitreous haemorrhage.
\end{abstract}

Keywords: Retinal Cavernous Haemangioma; Intravitreal Bevacizumab; Persistent Proliferative Diabetic Retinopathy; Vitreous Haemorrhage

\section{Introduction}

We would like to report a case of Retinal Cavernous Haemangioma treated with Intravitreal Bevacizumab, which was initially diagnosed as persistent proliferative diabetic retinopathy with multiple episodes of vitreous haemorrhage.

\section{Case}

A 59 year-old insulin dependent diabetic male was referred from another ophthalmic unit with a diagnosis of persistent retinal neovascularisation in the left eye, despite repeat sessions of laser photocoagulation. He had a history of multiple mild to moderate self-limiting vitreous haemorrhages in the past which settled without any surgical intervention. Previous medical history apart from diabetes mellitus was insignificant. On examination Best-Corrected Visual Acuity (BCVA) was logMAR $0.00 \mathrm{OD}$ and $0.18 \mathrm{OS}$. Anterior segment examination was unremarkable. Fundus examination showed a cluster of abnormal vessels in the juxtapapillary region of the temporal retina (Figure 1(a)). Fluorescein Angiography (FFA) showed saccular lesions with slow filling in the

\footnotetext{
"Corresponding author.
}

early phase and late hyperfluorescence with typical fluid levels within the saccules (Figure 1(b)).Optical Coherence Tomography (OCT) showed a bunch of "grape-like", nodular retinal lesions proliferating to the posterior hyaloid space (Figure 1(c)). The working diagnosis was Retinal Cavernous Haemangioma. MRI brain and orbit was then performed which didn't show any abnormalities. A decision was made to treat the patient with a view to shrink these lesions and prevent a recurrence of vitreous haemorrhage. The patient received monthly intravitreal injections of Bevacizumab $(1.25 \mathrm{mg} / 0.05 \mathrm{ml})$ for three months. At the last follow up, 22 months following treatment, BCVA remained logMAR 0.18 OS. The lesions showed demonstrable decrease in dimensions and no leakage on FFA (Figures 2). During that period the patient develop no further episodes of vitreous haemorrhage.

\section{Discussion}

Cavernous haemangiomas of the retina are believed to be rare congenital vascular hamartomas and may present commonly as an incidental finding or rarely as a cause of vitreous haemorrhage [1]. Histologically, these vascular malformations have normal endothelial cells and support- 


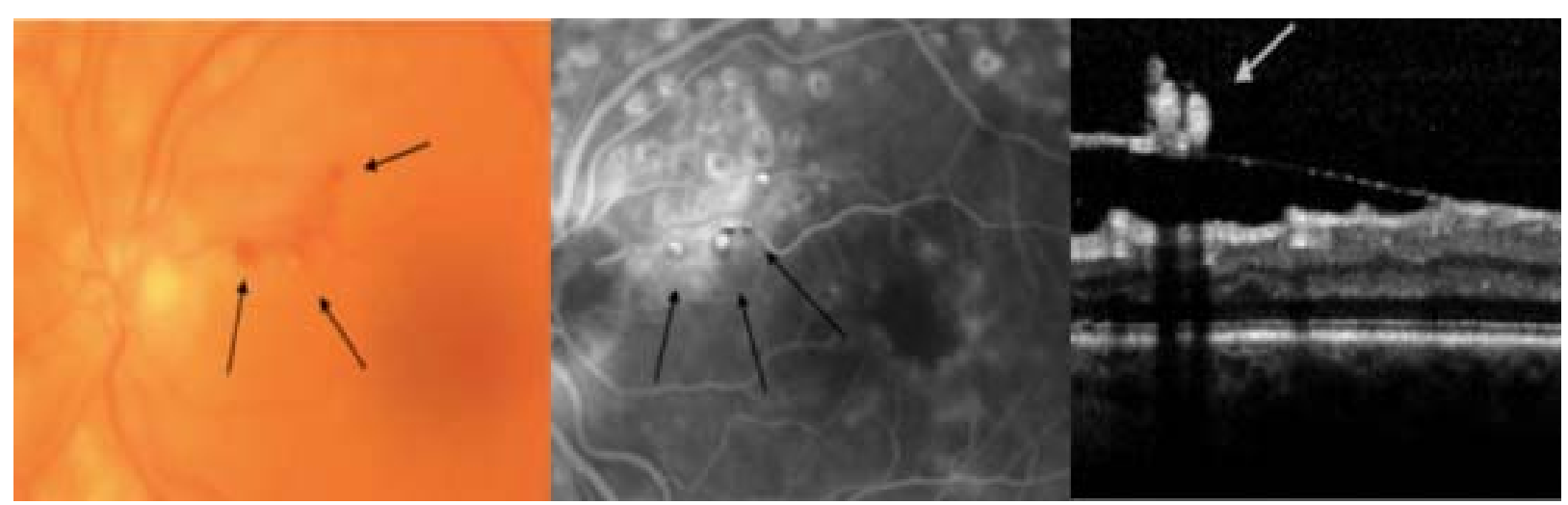

(a)

(b)

(c)

Figure 1. (a) Colour photo of left fundus showing "grape like" lesions temporal to the optic disc (arrows); (b) Fluorescein Angiogram in midphase showing leakage and hyperfluorescent menisci of the juxtapapillary lesions (arrows); (c) OCT showing retinal nodular lesions proliferating to the posterior hyaloid space (arrow).

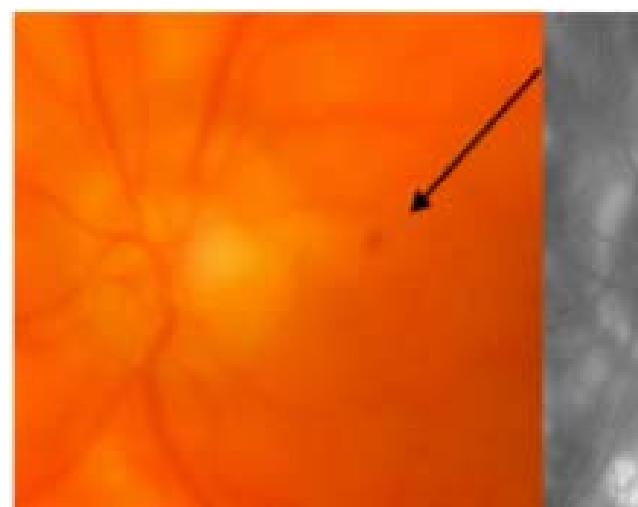

(a)

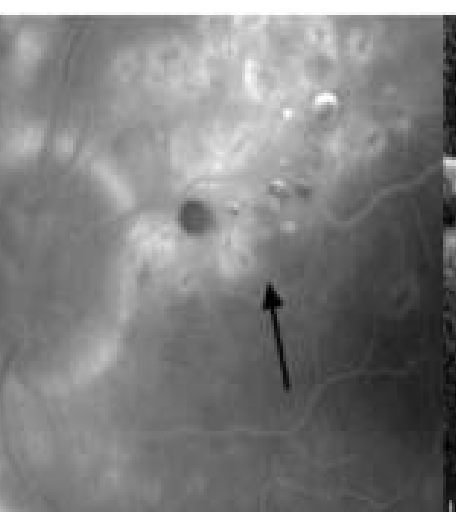

(b)

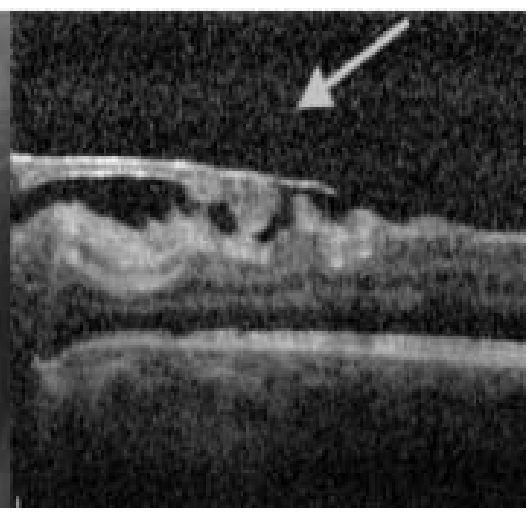

(c)

Figure 2. Twenty two months following treatment (a) Colour photo showing decrease in number and size of lesions (arrow); (b) Fluorescein Angiogram showing no leakage; (c) OCT showing no vascular proliferation to the posterior hyaloid space and significant shrinkage of lesions (arrow).

ing fibrous septa and therefore have less likelihood of leakage but may occasionally rupture and cause vitreous haemorrhage. They are often sporadic but familial bilateral cases have been reported [2]. In some patients, these tumours have been observed to coexist with intracranial cavernous haemangioma and angiomatous skin lesions $[3,4]$. It has been reported that $5 \%$ of the patients diagnosed with cerebral haemangioma have associated retinal cavernous haemangiomas [2]. Therefore the ophthalmologist plays a critical role in accurate and early diagnosis. Ophthalmoscopically, retinal cavernous haemangioma appears as a group of blood-filled saccules within the inner retinal layers. Relevant diagnostic tests include Fluorescein angiography and Optical coherence tomography (OCT). Patients may be asymptomatic in which case no treatment is necessary. However visual acuity can deteriorate due to accompanying epiretinal membrane or vitreous haemorrhage where vitrectomy may be needed [1]. In our patient the decision to treat the lesions with intravitreal Bevacizumab was taken in view of the risk of recurrent vitreous haemorrhage, as they were leaking on FFA. Following the treatment, the size and number of the retinal lesions reduced and the leakage disappeared with no recurrence of vitreous haemorrhage at last follow up.

\section{Conclusion}

In conclusion, we would like to stress the significance of identification of these lesions, which can be confused, with new vessels of diabetic retinopathy and lead to unnecessary photocoagulation. Furthermore, to the best of our knowledge this is the first case report where intravitreal Bevacizumab has been used for the treatment of retinal cavernous haemangioma.

\section{REFERENCES}

[1] E. Pringle, S. Chen, A. Rubinstein, C. K. Patel and S. Dow- 

Intravitreal Bevacizumab, Initially Labelled as Persistent Proliferative Diabetic Retinopathy

nes, "Optical Coherence Tomography in Retinal Cavernous Haemangioma may Explain the Mechanism of Vitreous Haemorrhage,” Eye, Vol. 23, 2009, pp. 1242-1243. doi:10.1038/eye.2008.156

[2] P. Labauge, V. Krivosic, C. Denier, E. Tounier-Lasserve and A. Gaudric, "Frequency of Retinal Cavernomas in 60 Patients with Familial Cerebral Cavernomas: A Clinical and Genetic Study," Archives of Ophthalmology, Vol. 124, No. 6, 2006, pp. 885-886. doi:10.1001/archopht.124.6.885

[3] H. Heimann and B. Damato, "Congenital Vascular Malformations of the Retina and Choroid," Eye, Vol. 24, No. 3, 2010, pp. 459-467. doi:10.1038/eye.2009.310

[4] A. D. Singh, P. A. Rundle and I. Rennie, "Retinal Vascular Tumors," Ophthalmology Clinics of North America, Vol. 18, No. 1, 2005, pp. 167-176. doi:10.1016/j.ohc.2004.07.005 